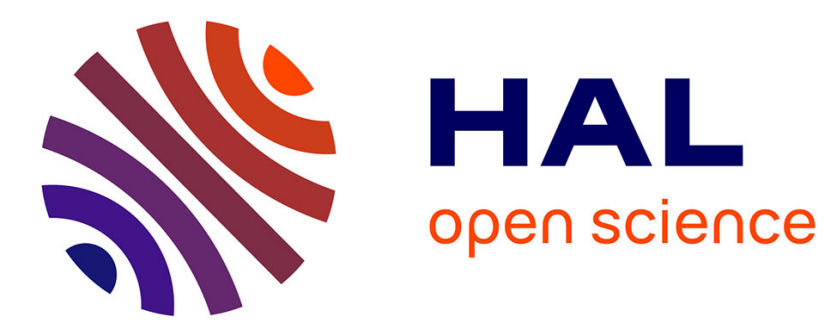

\title{
Traitement néoadjuvant des cancers du rectum
}

\author{
Jean-Baptiste Bachet, Stéphane Benoist, Léo Mas, Florence Huguet
}

\section{To cite this version:}

Jean-Baptiste Bachet, Stéphane Benoist, Léo Mas, Florence Huguet. Traitement néoadjuvant des cancers du rectum. Bulletin du Cancer, 2021, 10.1016/j.bulcan.2021.03.018 . hal-03266532

\section{HAL Id: hal-03266532 \\ https://hal.sorbonne-universite.fr/hal-03266532}

Submitted on 21 Jun 2021

HAL is a multi-disciplinary open access archive for the deposit and dissemination of scientific research documents, whether they are published or not. The documents may come from teaching and research institutions in France or abroad, or from public or private research centers.
L'archive ouverte pluridisciplinaire HAL, est destinée au dépôt et à la diffusion de documents scientifiques de niveau recherche, publiés ou non, émanant des établissements d'enseignement et de recherche français ou étrangers, des laboratoires publics ou privés. 


\section{Article de Synthèse}

Titre : traitement néoadjuvant des cancers du rectum

Title: neoadjuvant treatment for rectal cancer

Auteurs : Jean-Baptiste Bachet, ${ }^{1,2}$ Stéphane Benoist, ${ }^{3,4}$ Léo Mas, ${ }^{2}$ Florence Huguet ${ }^{1,5}$

\section{Affiliations :}

1. Sorbonne Université, UPMC Université, IUC, Paris France ; 2. Assistance Publique Hôpitaux de Paris, Service d'Hépato-gastroentérologie et Oncologie digestive, Groupe Hospitalier Pitié Salpêtrière, Paris, France ; 3. Assistance Publique - Hôpitaux de Paris, Service de Chirurgie Digestive et Oncologique, CHU Bicêtre, le Kremlin Bicêtre ; 4. Université Paris-Saclay, UFR médecine, Le kremlin Bicêtre ; 5. Assistance Publique Hôpitaux de Paris, Service d'Oncologie Radiothérapie, Hôpital Tenon, Paris, France

\section{Correspondance :}

Pr Jean-Baptiste Bachet

Service d'Hépato-gastroentérologie et Oncologie digestive, Groupe Hospitalier Pitié Salpêtrière, APHP, Paris, France

47-83 Boulevard de l'hôpital 75651 Paris Cedex 13

Tel: 0142161041 ; Fax: 0142161238

Mail : jean-baptiste.bachet@aphp.fr 


\section{Résumé}

La prise en charge des patients avec un cancer du rectum localement avancé s'est beaucoup améliorée ces dernières années avec la radiothérapie pré-opératoire (RT) et l'exérèse complète du mésorectum. Le taux de récidive locale est désormais de l'ordre de $5 \%$ mais le risque de récidive métastatique, de l'ordre de 30\%, n'avait pas été réduit jusqu'à présent. Le bénéfice de la chimiothérapie adjuvante reste discutable en dehors des patients avec une tumeur ypN+ après chimioradiothérapie (CRT) pré-opératoire pour lesquels le FOLFOX est une option.

Ces dernières années, plusieurs essais thérapeutiques ont évalué l'intérêt de l'allongement du délai entre la fin de la radiothérapie et la chirurgie et/ou le bénéfice d'une chimiothérapie néoadjuvante, administrée en induction (avant RT) ou en consolidation (après RT et avant chirurgie). Deux essais de phase 3, PRODIGE 23 et RAPIDO, dont les premiers résultats ont été rapportés en 2020 sont positifs sur leur critère de jugement principal. Les deux schémas évalués dans ces essais sont très différents mais ont permis de démontrer qu'une chimiothérapie néoadjuvante permettait de réduire significativement le risque métastatique.

Les développements actuels portent pour beaucoup sur une désescalade thérapeutique: conservation d'organe selon une stratégie de «Watch and Wait» ou résection locale de la cicatrice ou administration d'une chimiothérapie néoadjuvante sans radiothérapie. Ces stratégies thérapeutiques ne sont pas encore validées mais devraient faire l'actualité de demain. Cette revue a pour but de faire le point sur les données récentes rapportées chez les patients avec un cancer du rectum localement avancé. 


\section{Abstract}

The management of patients with locally advanced rectal cancer has improved significantly in the past few years with preoperative radiotherapy $(\mathrm{RT})$ and total mesorectal excision. The rate of local recurrence is now around $5 \%$ while the risk of metastatic recurrence has not been reduced which is about $30 \%$. The benefit of adjuvant chemotherapy remains questionable apart from patients with ypN + tumor after preoperative chemoradiotherapy (CRT) for whom FOLFOX is an option.

In recent years, several therapeutic trials have evaluated the benefit of extending the time between the end of RT and surgery and / or the benefit of neoadjuvant chemotherapy, administered as induction (before RT) or in consolidation (after RT and before surgery). The first results of two positive phase 3 trials, PRODIGE 23 and RAPIDO, have been reported in 2020. The two regimens evaluated in these trials are markedly different but have shown that neoadjuvant chemotherapy significantly reduces the risk of distant metastasis.

Current developments largely related to a de-escalation of therapy: organ conservation according to a "Watch and Wait" strategy or local resection of the scar, administration of neoadjuvant chemotherapy without RT. These therapeutic strategies have not yet been validated but should be in the news tomorrow.

The purpose of this review is to present recent data reported in patients with locally advanced rectal cancer. 
Mots-clés : cancer du rectum ; radiothérapie ; chimioradiothérapie ; chimiothérapie ; néoadjuvant ; réponse pathologique complète

Keywords: rectal cancer; radiation therapy; chemoradiotherapy; chemotherapy; neoadjuvant; complete pathological response 


\section{Introduction}

Les cancers du rectum représentent près d'un tiers des cancers colorectaux et sont l'un des cancers digestifs les plus fréquents. En cas de cancer du rectum localement avancé (T3-4 et/ou $\mathrm{N}+$ ), la prise en charge thérapeutique a longtemps reposé sur un schéma court de radiothérapie (SCRT : 25 Gy en 5 fractions et 5 jours) ou sur une chimioradiothérapie (CRT : 45 à 50 Gy en 5 fractions par semaine de 1,8 à 2 Gy avec 5-fluorouracile ou capécitabine concomitant) néoadjuvante suivie d'une proctectomie avec exérèse complète du mésorectum dans un délai de 7-10 jours ou 6-8 semaines, respectivement.[1-3] Contrairement à la CRT néoadjuvante, la SCRT n'est pas associée à une réduction de la taille tumorale et, en cas de risque de résection R1 par envahissement de la marge de résection circonférentielle, une CRT doit être préférée.[4] Cette stratégie thérapeutique a permis de réduire drastiquement le taux de récidive locale à 5\% environ mais n’a jamais permis de diminuer le risque de récidive métastatique.[1-3] Après une CRT comportant une fluoropyrimidine, le taux de réponse pathologique complète (RPc) est de l'ordre de 15\%.[3,5-7] L'obtention d'une RPc sur la pièce de résection chirurgicale est un facteur pronostique, associé à une diminution du risque de récidive locorégionale et métastatique, et à une augmentation des taux de survies sans récidive et globale.[8]

La chimiothérapie adjuvante après résection d'un cancer du rectum n'a jamais démontré d' intérêt dans une étude de phase 3 malgré un faisceau d'arguments concordants.[9-12] Dans l'étude de phase 2 ADORE, les patients étaient randomisés en post-opératoire selon le stade ypTNM (stade II ou III après CRT) entre un schéma FUFOL selon la Mayo Clinic et un FOLFOX4 simplifié pour une durée de 4 mois.[13] Les patients traités en adjuvant par FOLFOX4 avaient une survie sans récidive [HR=0,66; IC 95\%:0,43-0,99] et une survie globale [HR=0,46; IC 95\% : 0,21-0,97] significativement plus longues. Le bénéfice était essentiellement observé en cas de tumeur ypN+ et une chimiothérapie adjuvante par FOLFOX4 
est désormais considérée comme une option chez ces patients.[4] Tous les essais ayant évalué l'intérêt d'une chimiothérapie adjuvante ont permis de souligner la difficulté de l'administrer en post-opératoire avec, en intention de traiter (patients inclus avant la CRT), près de $30 \%$ à $50 \%$ des patients ne la débutant pas.[9,14-16]

Suite aux résultats du FOLFOX ou CAPOX en situation adjuvante chez les patients opérés d'un cancer du côlon, plusieurs essais thérapeutiques de phase 3 ont évalué l'intérêt de l'ajout de l'oxaliplatine durant la CRT néoadjuvante chez les patients ayant un cancer du rectum localement avancé.[14,17-20] Globalement, l'ajout de l'oxaliplatine durant la CRT était associé à une augmentation des toxicités et une moins bonne compliance à la CRT, et ne permettait d'augmenter ni le taux de RPc, ni les survies sans récidive ou globale. La seule étude ayant rapporté une augmentation significative du taux de RPc (17\% vs 13\%) et de la survie sans récidive (taux à 3 ans 76\% vs 71\%) était l'étude allemande CAO/ARO/AIO-04 dans laquelle le bras expérimental comportait l'ajout de l'oxaliplatine durant la CRT mais également durant la chimiothérapie adjuvante (FOLFOX vs 5FU).[14] Néanmoins, il n'existait pas non plus de différence de survie globale dans cette étude. Suite aux résultats de l'ensemble de ces études [14,17-20], l'ajout de l'oxaliplatine à la CRT n'est actuellement pas recommandé.[4]

La séquence SCRT ou CRT suivie de la résection rectale est associée à une mortalité de 1 à $2 \%$ augmentant avec l'âge et les comorbidités et, à long-terme, à des séquelles sur les fonctions sexuelles et urinaires dans près de $60 \%$ des cas.[21-25] Ces constatations ont amené à évaluer la possibilité de conserver le rectum chez les patients en réponse clinique complète $(\mathrm{RCc})$ après CRT.[26] Cette stratégie représente néanmoins un challenge important car si elle permet d'éviter une résection chirurgicale et ses séquelles potentielles chez certains patients, d'autres patients devront avoir une chirurgie de rattrapage en cas de récidive locorégionale durant le suivi. Cette chirurgie de rattrapage est associée à une morbidité élevée en raison de plans 
chirurgicaux perturbés, une dissection pelvienne difficile et un risque de lésions du plexus hypogastrique.[27,28]

Ces dernières années se sont développés de nouveaux schémas thérapeutiques consistant à ajouter à la CRT pré-opératoire une chimiothérapie néoadjuvante réalisant un traitement complet en pré-opératoire appelé « traitement néoadjuvant total ». Ce concept de traitement néoadjuvant total vient d'être évalué dans plusieurs essais récents et en particulier, deux essais de phase 3 dont les résultats ont été rapportés au congrès de l'ASCO 2020.[29,30]

L'objectif de cette revue de la littérature est de faire le point sur les données récentes rapportées chez les patients ayant un cancer du rectum localement avancé, sur les essais en cours, de discuter de potentiels nouveaux standards (en attendant une mise à jour des recommandations nationales et internationales) et des questions restant en suspens.

\section{Amélioration des résultats de la radiothérapie par allongement du délai entre la radiothérapie et la chirurgie et/ou l'ajout d'une chimiothérapie néoadjuvante}

La RPc ne peut pas être un critère de substitution de la survie sans récidive ou globale mais est un facteur pronostique majeur et permet théoriquement d'envisager une stratégie de conservation d'organe.[31,32] Pour ces raisons, plusieurs stratégies thérapeutiques visant à augmenter le taux de RPc ont été évaluées ces dernières années. Celles-ci reposent principalement sur l'allongement du délai entre la fin de la radiothérapie et la chirurgie et/ou sur l'ajout d'une chimiothérapie néoadjuvante en induction (avant la radiothérapie) ou en consolidation (après la radiothérapie et avant la chirurgie). Les principaux résultats de ces essais sont détaillés dans le tableau 1. 
L'essai de phase 3 Stockholm III a comparé trois bras de traitement : un bras SCRT «standard » avec chirurgie 7 jours après, un bras SCRT avec une chirurgie décalée entre 4 à 8 semaines après la fin de la radiothérapie et un bras de radiothérapie normofractionnée de 50 Gy avec une chirurgie 4 à 8 semaines après. En ce qui concerne les 2 bras de SCRT, l'allongement du délai entre la fin de la SCRT et la chirurgie était associé à une augmentation significative du taux de RPc, passant de $<1 \%$ à 1 semaine à $10 \%$ après $4-8$ semaines d'attente $(\mathrm{p}<0,0001)$.[33,34] Néanmoins, bien qu'une RPc soit un facteur pronostique indépendant,[34] les taux de récidive locorégionale ou métastatique, et les taux de survie sans récidive et globale étaient similaires entre les trois bras.[33] L'allongement du délai après SCRT était associé à une augmentation significative des hospitalisations pour toxicité aiguë liée à la radiothérapie (respectivement, 7\% vs $<1 \%, \mathrm{p}<0,0001$ ) mais à une diminution significative de la morbidité chirurgicale (taux de complications post-opératoires $41 \%$ vs $53 \%, \mathrm{p}=0,001)$.[33]

L'essai de phase 3 Polish II a lui comparé deux bras de traitement : un bras contrôle avec une CRT (50,4 Gy, capécitabine + oxaliplatine) suivie d'une chirurgie à 6-8 semaines et un bras expérimental comportant une SCRT suivie de 3 cycles de FOLFOX en consolidation puis une chirurgie à 11 semaines de la fin de la radiothérapie.[35,36] Les taux de résection R0 (71\% vs $77 \%)$ et de RPc (12\% vs 16\%) étaient plus élevés dans le bras SCRT + consolidation mais de manière non significative.[35] Après une médiane de suivi de 7 ans, il n'existait pas de différence entre les deux bras pour les taux de récidive locorégionale ou métastatique, et les taux de survie sans récidive et globale.[36] Le taux de toxicité aiguë préopératoire était significativement moins élevé dans le bras expérimental (toutes toxicités $83 \%$ vs $75 \%$, p $=0,006$ ) mais il n'existait pas de différence entre les deux bras pour les toxicités de grade 3-4 ou les décès liés au traitement.[35] Les complications post-opératoires et les complications tardives n'étaient pas différentes entre les deux bras.[35,36] 
Plusieurs études rétrospectives avaient suggéré qu'un allongement du délai avant chirurgie après CRT pourrait être associé à une augmentation du taux de RPc. Cette hypothèse a été infirmée par les résultats de l'essai de phase 3 GRECCAR-6 qui a comparé un bras standard avec une résection chirurgicale réalisée 7 semaines après la CRT et un bras expérimental avec une résection 11 semaines après.[37] Les taux de RPc étaient de 15\% dans le bras 7 semaines et de $17 \%$ dans le bras 11 semaines $(\mathrm{p}=0,60)$. Après un suivi de 32 mois, il n'existait pas de différence entre les deux bras pour les taux de récidive locorégionale ou métastatique, et les taux de survie sans récidive et globale.[38] L'allongement du délai après CRT était par contre associé à une augmentation de la morbidité post-opératoire $(44,5 \% \mathrm{vs} 32 \%, \mathrm{p}=0,04)$ et à une moins bonne qualité de résection complète du mésorectum $(79 \%$ vs $90 \%, \mathrm{p}=0,016)$.[38] Une chimiothérapie d'induction à base de FOLFOX, CAPOX ou FOLFIRINOX a démontré son efficacité sur la tumeur primitive rectale avec un contrôle tumoral dans plus de $95 \%$ des cas, des réponses objectives (selon RECIST au scanner ou selon le volume tumoral en IRM) et une amélioration rapide des symptômes rectaux chez plus de 90\% des patients.[39-41] Plusieurs études de phase 2 ont évalué une chimiothérapie d'induction avant CRT (tableau 1).[41-45] La chimiothérapie d'induction consistait le plus souvent en du CAPOX ou FOLFOX ; le nombre de cycles en induction était variable (de 1 à 4) et la CRT pouvait comporter de l'oxaliplatine en concomitant. Dans ces études, en intention de traiter, le taux de RPc variait de $14 \%$ à $25 \%$. Dans l'étude de phase 2 randomisée GCR-3, une chimiothérapie de type CAPOX (4 cycles) était mieux tolérée et associée à une dose intensité plus élevée lorsqu'elle était administrée en induction par rapport à une administration en adjuvant.[43] Néanmoins, les taux de résection R0 et de RPc ainsi que les survies sans récidive et globale à long terme n'étaient pas augmentés par la chimiothérapie d'induction.[43,46] 
La chimiothérapie néoadjuvante peut également être administrée en consolidation, après la CRT et avant la chirurgie, plutôt qu'en induction. Deux études de phase 2 randomisées ont évalué une telle stratégie.[45,47] Dans celles-ci, la chimiothérapie de consolidation utilisée était du FOLFOX, avec un nombre de cycles variables, allant de 2 à 6 cycles selon les bras. L'administration de la chimiothérapie de consolidation était associée à un allongement du délai entre la fin de la CRT et la chirurgie, délai augmentant en fonction du nombre de cycles administrés (tableau 1). Par rapport à la CRT seule, l'administration d'une chimiothérapie de consolidation était associée à une augmentation significative du taux de RPc : $25 \%$ pour 2 cycles, $30 \%$ pour 4 cycles et $38 \%$ pour 6 cycles $(p=0,0036)$.[47] Le taux d'effets secondaires de grade 3-4 sous FOLFOX augmentait en fonction du nombre de cycles reçus mais la difficulté chirurgicale et les taux de complications post-opératoires n'étaient pas différents entres les bras.[47] L'étude de phase $2 \mathrm{CAO/ARO/AIO-12}$ a comparé une chimiothérapie néoadjuvante par 3 cycles de FOLFOX administrés en induction ou en consolidation.[45] La CRT était associée à moins de toxicité de grade 3-4 (37\% vs 27\%) et une meilleure compliance dans le bras consolidation que dans le bras induction. De plus, le taux de RPc était de $25 \%$ dans le bras consolidation versus $17 \%$ en induction, et seul le bras consolidation atteignait l'hypothèse statistique prédéfinie.[45]. En d'autres termes, l'ensemble de ces études semble montrer qu'en cas d'ajout d'une chimiothérapie néoadjuvante à une CRT, son administration en consolidation permet d'obtenir plus de réponses tumorales qu'en induction avec une meilleure tolérance de la CRT.

\section{Deux potentiels nouveaux standards !}

Deux essais de phases 3 randomisés ont été présentés à l'ASCO 2020.[29,30] Ces deux études ont évalué deux schémas expérimentaux différents mais avaient le même bras contrôle, à savoir 
une CRT (50,4 Gy, capécitabine). L'étude PRODIGE 23 a évalué une chimiothérapie d'induction par FOLFIRINOX (6 cycles) (Figure 1) alors que l'étude RAPIDO a évalué un schéma de SCRT suivi d'une chimiothérapie de consolidation par CAPOX (6 cycles) ou FOLFOX (9 cycles) (Figure 2).

Les principales caractéristiques des patients inclus dans ces essais ainsi que les traitements reçus en intention de traiter sont résumés dans le tableau 2. Les patients inclus dans l'étude PRODIGE 23 devaient avoir un cancer du rectum localement avancé (T3-4 et/ou N+). Cette étude a été menée exclusivement en France.[29] Les patients inclus dans l'étude RAPIDO devaient avoir un cancer du rectum localement avancé défini comme à haut risque par la présence d'au moins un des critères suivants : $\mathrm{T} 4 \mathrm{a} / \mathrm{b}$, invasion vasculaire extramurale, $\mathrm{N} 2$, envahissement du fascia du mésorectum, et/ou ganglions lymphatiques latéraux hypertrophiés. Cette étude était internationale et la majorité des patients ont été inclus aux Pays Bas (39\%), en Suède (36\%) et en Espagne (13\%).[30] Les principaux résultats des deux essais sont détaillés dans le tableau 3. Dans l'essai PRODIGE 23, 90\% des patients du bras expérimental ont reçu les 6 cycles planifiés de FOLFIRINOX avec un profil de tolérance acceptable. Parmi les patients ayant reçu la CRT, la dose de radiothérapie reçue n'était pas différente entre les deux bras mais le taux d'interruption de la capécitabine était plus élevé dans le bras expérimental ( $8,3 \%$ vs 3,1\% ; $\mathrm{p}<0,02)$ de même que le taux de neutropénie de grade $3-4(2,8 \%$ vs $0 \% ; \mathrm{p}<0,02)$.[29] Au bilan d'évaluation après traitement néoadjuvant, 8 patients du bras contrôle versus aucun du bras expérimental $(p=0,007)$ n'avaient pu être opérés en raison d'une tumeur non résécable $(n=3)$ ou de métastases $(n=5)$. La morbidité post-opératoire, les taux de ré-hospitalisation et de réintervention et la mortalité post-opératoire n'étaient pas différents entre les deux bras en dehors de la mortalité dans les 60 jours post-opératoires significativement plus importante dans le bras contrôle $(2,8 \%$ vs $0 \% ; p=0,03)$. La chimiothérapie adjuvante était significativement mieux tolérée dans le bras expérimental et la dose intensité reçue significativement supérieure que 
dans le bras contrôle. Il n'existait pas de différence entre les deux bras pour la qualité de vie.[29] Après une médiane de suivi de 46,5 mois, le taux de survie sans maladie à 3 ans qui était le critère principal de jugement $(\mathrm{HR}=0,69$; IC 95\% : 0,49-0,97; $\mathrm{p}=0,034)$ et le taux de survie sans métastase à 3 ans $(\mathrm{HR}=0,64 ; \mathrm{IC} 95 \%: 0,44-0,93 ; \mathrm{p}=0,017)$ étaient significativement allongés dans le bras expérimental (tableau 3). En analyse multivariée, le bras expérimental était associé à une augmentation significative du taux de survie sans maladie $(\mathrm{HR}=0,68$; IC $95 \%: 0,48-0,97 ; p=0,03)$.

Dans l'essai RAPIDO, la très large majorité des patients du bras expérimental ont reçu le CAPOX en consolidation après radiothérapie et avant chirurgie comme planifié $(99 \%$, $\mathrm{n}=454 / 460) ; 44 \%$ des patients avaient eu une réduction de dose et $85 \%$ avaient reçu tous les cycles planifiés.[30] Durant les traitements préopératoires, les taux d'effets secondaires de grade $\geq 3$ étaient de $48 \%$ dans le bras expérimental et de $25 \%$ dans le bras contrôle.[48] Au bilan d'évaluation après traitement néoadjuvant, 3 patients du bras expérimental et 2 du bras contrôle n'avaient pas été opérés en raison d'une progression métastatique. Dans le bras expérimental, les pertes sanguines durant la chirurgie et la durée d'hospitalisation étaient significativement plus élevées que dans le bras contrôle alors que le taux de résection complète du mésorectum était significativement inférieur (78\% vs $85 \% ; p=0,032)$.[48] Néanmoins, il n'existait pas de différence significative entre les deux bras pour le type de chirurgie réalisée ou les complications post-opératoires. $42 \%$ des patients du bras contrôle avaient reçu une chimiothérapie adjuvante; le taux d'effets secondaires de grade $\geq 3$ était de $35 \%$ et le taux d'interruption de traitement de 37\%.[30] Après une médiane de suivi de 55,2 mois, le taux de survie sans échec du traitement lié à la maladie à 3 ans qui était le critère principal de jugement $(\mathrm{HR}=0,75 ; \mathrm{IC} 95 \%: 0,60-0,95 ; \mathrm{p}=0,019)$ et le taux de survie sans métastase à 3 ans $(\mathrm{HR}=0,69$ ; IC 95\% : 0,54-0,90; $\mathrm{p}=0,0048$ ) étaient significativement allongés dans le bras expérimental (Tableau 3). 
Ces deux études de phase 3 sont donc positives pour leur objectif principal avec des résultats oncologiques très similaires. Elles démontrent qu'une chimiothérapie néoadjuvante, en induction ou en consolidation, permet de réduire significativement le risque de récidive métastatique. Ces deux schémas semblent tolérables avec des profils de toxicités connus. Dans l'essai RAPIDO, le taux élevé d'amputation abdominopérinéale dans les deux bras de traitement, s'explique principalement d'une part par le fait que les tumeurs étaient initialement localement très évoluées mais également par des habitudes chirurgicales nationales. Les données de l'étude RAPIDO suggère néanmoins que la chirurgie pourrait être plus difficile après SCRT et une chimiothérapie de consolidation de 18 semaines comme l'atteste les taux de conversion en laparotomie, de pertes sanguines et de plaie du mésorectum qui étaient plus élevés dans le bras expérimental.[48] Les taux de récidive locale étaient de 4,8\% et 8,3\% dans les bras expérimentaux de PRODIGE 23 et RAPIDO, respectivement, versus $7,0 \%$ et $6,0 \%$ dans les bras contrôles respectifs.[29,30] Des données plus matures sont nécessaires pour évaluer le bénéfice de ces schémas sur la survie globale. Ces deux nouveaux schémas thérapeutiques n'en constituent pas moins deux nouveaux standards potentiels. Les analyses de sous-groupes avec Forrest Plot n'ont pour l'instant pas été rapportées pour ces deux études et il n'existe pour l'instant pas d'élément formel permettant de privilégier l'un ou l'autre schéma, que ce soit selon la localisation tumorale ou le stade TNM initial.Tous les patients ne sont pas éligibles à recevoir du FOLFIRINOX en induction et l'indication de l'un ou l'autre schéma thérapeutique sera à discuter en réunion de concertation pluridisciplinaire selon les caractéristiques des patients (âge, comorbidités).

\section{Futures stratégies de désescalade}


En raison du faible taux de récidive locale et de taux de RCc et RPc non négligeables, plusieurs stratégies thérapeutiques évaluent actuellement la possibilité d'une désescalade thérapeutique chez des patients sélectionnés. Cette désescalade peut avoir pour but d'éviter une chirurgie rectale, de limiter la chirurgie rectale ou de s'affranchir de la radiothérapie.

\section{Stratégie de "Watch and Wait »}

Une stratégie de conservation rectale des patients en RCc après CRT avec une surveillance rapprochée (stratégie dite de «Watch and Wait») a été développée par l'équipe de Habr-Gama et al au Brésil.[26] Les données rapportées par cette équipe sont très encourageantes avec peu de récidive locale et des taux de survie sans récidive et globale à 5 ans tout à fait acceptables. Néanmoins, ces données sont peu reproductibles et plusieurs autres séries ont rapporté des taux de récidive locorégionale nettement plus élevés.[49] Ces différences sont probablement expliquées par l'hétérogénéité des stades initiaux des tumeurs, des traitements administrés en néoadjuvant, des critères d'évaluation de la RCc et des modalités de surveillance. Dans le but de générer des données plus robustes, un registre international (15 pays participants) de « Watch and Wait » a été mis en place. Des données sur 880 patients en RCc inclus dans ce registre ont été rapportées.[50] Après un suivi médian de 39,6 mois, le taux de récidive locorégionale à 2 ans était de 25,2\%. La plupart (88\%) de ces récidives locorégionales survenaient durant les deux premières années et $97 \%$ étaient localisées dans la paroi rectale. Le taux de progression métastatique était de $7 \%$, et les taux de survie spécifique et de survie globale à 5 ans étaient de $94 \%$ et $85 \%$, respectivement. Le recueil de données ayant été en partie rétrospectif les mêmes hétérogénéités entre les centres que celles précédemment décrites étaient observées.[49,50]. Ce taux de récidive locale de plus de $20 \%$ a été confirmé par une méta-analyse portant sur 692 patients publiée la même année.[51] Des données prospectives sur des séries plus homogènes 
de patients sont nécessaires. La définition d'une RCc doit se baser sur les résultats du toucher rectal, de la rectoscopie et de 1'IRM.[4]

L'étude de phase 2 randomisée OPRA a comparé deux bras de traitement néoadjuvant avec une CRT (50-56 Gy, capécitabine) associée à une chimiothérapie en induction ou en consolidation (CAPOX ou FOLFOX 4 mois). Les patients étaient réévalués à la fin du traitement néoadjuvant et une stratégie de «Watch and Wait » était proposée à ceux en RCc. Des résultats préliminaires ont été rapportés à l'ASCO 2020 après un suivi médian de 27,1 mois.[52] Les taux de survie sans maladie ( $78 \%$ et $77 \%$ ) et sans métastase ( $84 \%$ et $82 \%)$ à 3 ans étaient identiques dans les deux bras consolidation et induction, respectivement. Par contre, le taux de survie sans exérèse du mésorectum à 3 ans était supérieur dans le bras consolidation (59\% vs 43\%).[52] Des résultats plus matures sont nécessaires avant toute conclusion définitive mais ces résultats sont relativement concordants avec ceux de l'étude $\mathrm{CAO} / \mathrm{ARO} / \mathrm{AIO}-12$ dans laquelle le bras consolidation était associé à un taux de RPc plus élevé que le bras induction.[47] Ce bras consolidation est en cours d'évaluation dans une étude de phase 2 de conservation d'organe (CAO/ARO/AIO-16) et dans une étude de phase 3 randomisée versus CRT (CAO/ARO/AIO18).

\section{Stratégie d'exérèse locale}

En dehors de la stratégie de « Watch and Wait » qui ne comporte aucune résection, une stratégie de résection locale de la cicatrice pourrait aussi permettre une conservation d'organe. La faisabilité de cette stratégie après CRT a été démontrée dans deux études de phase 2 ayant inclus des tumeurs de petite taille (cT2N0 et de moins de $4 \mathrm{~cm}$ ou cT1-3N0 et de moins de 5 cm).[53,54] L'essai de phase 3 GRECCAR-2 a inclus des patients avec un cancer du rectum de stade cT2-3Nx et une taille tumorale maximale de $4 \mathrm{~cm}$.[28] Après CRT (50 Gy, capécitabine +/- oxaliplatine), les patients répondeurs à l'IRM avec une tumeur résiduelle de moins de $2 \mathrm{~cm}$ 
étaient randomisés entre un bras exérèse locale $(n=74)$ et un bras chirurgie avec exérèse du mésorectum $(\mathrm{n}=71)$. Parmi les 74 patients ayant eu une résection de la cicatrice, 26 patients (35\%) avaient une tumeur ypT2 ou ypT3 et eurent une chirurgie complémentaire avec exérèse du mésorectum. Le critère de jugement principal était un critère composite comprenant les décès, les récidives, la morbidité et les effets secondaires. Les taux de survenue à 2 ans d'au moins un des évènements du critère composite étaient de $56 \%$ dans le bras exérèse locale et de 48\% dans le bras chirurgie radicale $(\mathrm{OR}=1,33 ; \mathrm{IC} 95 \%: 0,62-2,86 ; \mathrm{p}=0,43)$.[28] La morbidité chirurgicale et les effets secondaires étaient significativement majorés en cas de chirurgie complémentaire avec exérèse du mésorectum. Le taux de $\mathrm{N}+$ en cas de tumeur ypT0-1 était nul dans le bras chirurgie radicale. Après une médiane de suivi de 60 mois, les taux à 5 ans de récidive locorégionale ( $7 \%$ vs $7 \% ; p=0,60)$, de métastases $(18 \%$ vs $19 \% ; p=0,73)$, de survie sans maladie ( $70 \%$ vs $72 \% ; p=0,68)$, de survie globale $(84 \%$ vs $82 \% ; p=0,92)$ et de survie cancer-spécifique $(7 \%$ vs $10 \% ; \mathrm{p}=0,65)$ étaient identiques dans les bras exérèse locale et chirurgie radicale, respectivement.[55] Même si cet essai est négatif sur son critère principal composite, les résultats carcinologiques à long terme rapportés soutiennent l'intérêt de cette stratégie pour les patients ayant des petites tumeurs de moins de $4 \mathrm{~cm}$ avant traitement.

Deux autres essais dont les résultats sont attendus prochainement sont en cours. L'essai de phase 3 GRECCAR-12 fait suite à l'essai GRECCAR-2 et compare deux bras de traitement néoadjuvant : un bras de CRT seule et un bras de FOLFIRINOX 4 cycles suivi d'une CRT. Le but de cet essai est d'évaluer si une intensification du traitement pré-opératoire permet de réaliser une conservation d'organe à un plus grand nombre de patient. Les patients inclus doivent avoir au diagnostic une tumeur de stade cT2-3Nx et de moins de $4 \mathrm{~cm}$. La réponse tumorale est évaluée cliniquement et à l'IRM, et comme dans l'essai GRECCAR-2, une résection locale est proposée aux patients bon répondeurs. Le critère de jugement principal est le taux de conservation d'organe à 2 ans. L'essai de phase 3 OPERA compare lui deux bras de 
traitement néoadjuvant en augmentant la dose de radiothérapie : un bras de CRT (45 Gy, capécitabine) + boost externe de 9 Gy et un bras de CRT (45 Gy, capécitabine) + irradiation endocavitaire de 90 Gy en 3 fractions utilisant une radiothérapie de contact (RX 50KV). Les patients inclus doivent avoir au diagnostic une tumeur de stade cT2-3Nx et de moins de $5 \mathrm{~cm}$. En cas de réponse clinique complète, une exérèse locale ou une surveillance sont proposées. Le critère de jugement principal est le taux de préservation d'organe à 3 ans.

Actuellement les stratégies de conservation d'organe (WW ou exérèse locale) ne sont validées que pour des petites tumeurs cT2-T3 de petite taille ayant bien répondu à un traitement néoadjuvant plus ou moins intensifié. Le problème majeur de ces stratégies est le contingent de patients chez qui cette stratégie ne peut être menée à terme avec un risque plus élevé de séquelles liées à la CRT et de stomie définitive alors que leur tumeur initiale pouvait être traitée par une chirurgie conservant le sphincter sans irradiation préalable.

Pour des lésions plus évoluées initialement, même en cas de réponse complète et quasi complète, les taux de récidive locale après conservation sont encore trop élevés pour que cette stratégie puisse être un standard mais elle peut néanmoins être discutée au cas par cas chez des patients à haut risque chirurgical.

\section{Surveillance en cas de conservation d'organe}

Que ce soit dans le cadre d'une stratégie de WW ou d'exérèse locale, la surveillance du rectum est l'un des enjeux majeurs des stratégies de conservation d'organe. Cette surveillance est pour l'instant mal codifiée. Elle repose principalement sur l'examen clinique avec toucher rectal, l'IRM pelvienne et l'échoendoscopie rectale. A titre d'exemple, la surveillance proposée dans l'essai de phase 3 randomisée GRECCAR 12 en cas de conservation d'organe comporte un examen clinique, un scanner thoraco-abdomino-pelvien, une échoendoscopie rectale $+/-$ une IRM pelvienne, tous les 4 mois pendant 2 ans puis tous les 6 mois pendant 1 an. 
Dans le registre international de WW, et après une médiane de suivi de 55,2 mois, les patients sans récidive à 1 an avaient des taux de récidive locale ou à distance ultérieure de $11,9 \%$ et 6,2\%, respectivement.[56] Chez les patients sans récidive à 3 ans, ces taux étaient beaucoup plus faibles, de $2,7 \%$ et $2,2 \%$ respectivement. Ces données montrent que le risque de récidive est surtout important durant les $3^{\text {ères }}$ années mais qu'au-delà, le risque est limité.

\section{Traitement néoadjuvant sans radiothérapie}

Les progrès de ces dernières années ont permis de réduire drastiquement le taux de récidive locorégionale mais la CRT néoadjuvante est associée à un risque de toxicité à long terme sur les fonctions intestinales et génito-urinaires.[21,22] La chimiothérapie néoadjuvante a démontré son efficacité sur la tumeur primitive rectale, que ce soit pour le contrôle des symptômes ou la réduction de la taille tumorale.[39-41] Plusieurs essais ont évalué la possibilité de ne réaliser qu'une chimiothérapie néoadjuvante (FOLFOX le plus souvent) et de se passer de la radiothérapie.[40,57,58] Cette stratégie apparait faisable et intéressante avec chez les patients opérés des taux de résection $\mathrm{R} 0$ de $89 \%$ à $100 \%$ et des taux de réponse complète de $6,6 \%$ à $25 \%$. Un seul essai de phase 3 publié à ce jour a comparé un bras de chimiothérapie néoadjuvante (FOLFOX 4 à 6 cycles) seule à deux bras comportant une CRT avec LV5FU2 (5 cycles) ou FOLFOX (7 cycles).[58] Après un suivi médian de 45,2 mois, les résultats oncologiques à 3 ans étaient identiques avec, respectivement, des taux à 3 ans de survie sans maladie de $73,5 \%, 72,9 \%$ et $77,2 \%(\mathrm{p}=0,71)$, de récidive locorégionale de $8,3 \%, 8,0 \%$ et $7,0 \%$ $(p=0,87)$ et de survie globale de $90,7 \%, 91,3 \%$ et $89,1 \%,(p=0,97)$.

L'essai de phase 3 NORAD01 actuellement ouvert en France évalue une chimiothérapie néoadjuvante par FOLFIRINOX 6 cycles avant chirurgie. L'essai a été amendé suite aux résultats de l'essai PRODIGE23 et le bras de référence (antérieurement CRT seule) est désormais la séquence FOLFIRINOX 6 cycles suivi de CRT. Pour être inclus, les patients 
doivent avoir au diagnostic une tumeur du rectum de stade cT1-3N+ ou cT3N0 avec une marge de résection circonférentielle $>2 \mathrm{~mm}$ à l'IRM. Le critère de jugement principal est le taux de survie sans progression à 3 ans.

L'essai de phase 2-3 PROSPECT compare actuellement une chimiothérapie néoadjuvante par FOLFOX 6 cycles à la CRT chez les patients avec un cancer du rectum localement évolué. Dans le bras FOLFOX, une CRT est réalisée après les 6 cycles de FOLFOX si la tumeur n'a pas diminué de plus de 20\% (IRM ou échoendoscopie). Pour être inclus, les patients doivent avoir au diagnostic une tumeur du rectum de stade cT2-3N+ ou cT3N0 avec une marge de résection circonférentielle non envahie à l'IRM. Le critère de jugement principal de la phase 3 est la survie sans récidive.

\section{Conclusion}

Les résultats de plusieurs essais récents ont permis d'avancer dans la prise en charge thérapeutique des patients avec un cancer du rectum localement avancé. La chimiothérapie néoadjuvante utilisée en induction (comme dans PRODIGE 23) ou en consolidation (comme dans RAPIDO) a démontré son efficacité pour réduire le risque de récidive métastatique. Bien que les données de survie globale ne soient pas encore matures, cette réduction du risque de métastase justifie que ces deux protocoles soient considérés comme de nouveaux standards thérapeutiques. Néanmoins, tous les patients ne peuvent recevoir du FOLFIRINOX en induction et le schéma RAPIDO pourrait être privilégié chez des patients âgés ou avec comorbidités. Concernant les stratégies de conservation d'organe, le niveau de preuve reste actuellement insuffisant pour en faire un standard thérapeutique pour toutes les tumeurs en réponse clinique complète. Les patients doivent au mieux être inclus dans des essais thérapeutiques et ces stratégies doivent être réservées à des centres experts. 


\section{Déclaration de liens d'intérêt}

JB Bachet a perçu des honoraires (consultant, présentations) de la part des laboratoires Amgen, AstraZeneca, Bayer, Merck Serono, Pierre Fabre, Roche, Sanofi, Servier et Shire, et a reçu des financements de recherche des laboratoires Amgen, Merck Serono et Roche.

S Benoit a perçu des honoraires (consultant, présentations) de la part des laboratoires Amgen, Biom’up, Win'up surgical system, Merck Serono, Roche, Nestlé, Takeda, Abbvie, Biom'up, Sanofi, et Servier.

L Mas n'a pas de liens d'intérêt à déclarer.

F Huguet a perçu des honoraires (consultante, présentations) de la part des laboratoires Astra Zeneca, BMS, Merck Serono, MSD, Servier et Shire. 


\section{Références}

1. Peeters KCMJ, Marijnen CAM, Nagtegaal ID, Kranenbarg EK, Putter H, Wiggers T, et al. The TME trial after a median follow-up of 6 years: increased local control but no survival benefit in irradiated patients with resectable rectal carcinoma. Ann Surg 2007;246(5):693-701.

2. Quirke P, Steele R, Monson J, Grieve R, Khanna S, Couture J, et al. Effect of the plane of surgery achieved on local recurrence in patients with operable rectal cancer: a prospective study using data from the MRC CR07 and NCIC-CTG CO16 randomised clinical trial. Lancet 2009;373(9666):821-8.

3. Sauer R, Becker H, Hohenberger W, Rödel C, Wittekind C, Fietkau R, et al. Preoperative versus postoperative chemoradiotherapy for rectal cancer. N Engl J Med 2004;351(17):173140 .

4. Glynne-Jones R, Wyrwicz L, Tiret E, Brown G, Rödel C, Cervantes A, et al. Rectal cancer: ESMO Clinical Practice Guidelines for diagnosis, treatment and follow-up. Ann Oncol 2017;28(suppl_4):iv22-iv40.

5. Bosset JF, Collette L, Calais G, Mineur L, Maingon P, Radosevic-Jelic L, et al. Chemotherapy with preoperative radiotherapy in rectal cancer. $N$ Engl $J$ Med 2006;355(11):1114-23.

6. Gérard JP, Conroy T, Bonnetain F, Bouché O, Chapet O, Closon-Dejardin MT, et al. Preoperative radiotherapy with or without concurrent fluorouracil and leucovorin in T3-4 rectal cancers: results of FFCD 9203. J Clin Oncol 2006;24(28):4620-5.

7. Braendengen M, Tveit KM, Berglund A, Birkemeyer E, Frykholm G, Påhlman L, et al. Randomized phase III study comparing preoperative radiotherapy with chemoradiotherapy in nonresectable rectal cancer. J Clin Oncol 2008;26(22):3687-94.

8. Maas M, Nelemans PJ, Valentini V, Das P, Rödel C, Kuo LJ,et al. Long-term outcome in patients with a pathological complete response after chemoradiation for rectal cancer: a pooled analysis of individual patient data. Lancet Oncol 2010;11(9):835-44.

9. Bachet JB, Rougier P, de Gramont A, André T. [Rectal cancer and adjuvant chemotherapy: which conclusions?]. Bull Cancer 2010;97(1):107-22.

10. Breugom AJ, van Gijn W, Muller EW, Berglund Å, van den Broek CBM, Fokstuen T, et al. Adjuvant chemotherapy for rectal cancer patients treated with preoperative (chemo)radiotherapy and total mesorectal excision: a Dutch Colorectal Cancer Group (DCCG) randomized phase III trial. Ann Oncol 2015;26(4):696-701.

11. Glynne-Jones R, Counsell N, Quirke P, Mortensen N, Maraveyas A, Meadows HM, et al. Chronicle: results of a randomised phase III trial in locally advanced rectal cancer after neoadjuvant chemoradiation randomising postoperative adjuvant capecitabine plus oxaliplatin (XELOX) versus control. Ann Oncol 2014;25(7):1356-1362. 
12. Breugom AJ, Swets M, Bosset JF, Collette L, Sainato A, Cionini L, et al. Adjuvant chemotherapy after preoperative (chemo)radiotherapy and surgery for patients with rectal cancer: a systematic review and meta-analysis of individual patient data. Lancet Oncol 2015;16(2):200-7.

13. Hong YS, Nam BH, Kim KP, Kim JE, Park SJ, Park YS, et al. Oxaliplatin, fluorouracil, and leucovorin versus fluorouracil and leucovorin as adjuvant chemotherapy for locally advanced rectal cancer after preoperative chemoradiotherapy (ADORE): an open-label, multicentre, phase 2, randomised controlled trial. Lancet Oncol 2014;15(11):1245-53.

14. Rödel C, Graeven U, Fietkau R, Hohenberger W, Hothorn T, Arnold D, et al. Oxaliplatin added to fluorouracil-based preoperative chemoradiotherapy and postoperative chemotherapy of locally advanced rectal cancer (the German CAO/ARO/AIO-04 study): final results of the multicentre, open-label, randomised, phase 3 trial. Lancet Oncol 2015;16(8):979-89.

15. Sainato A, Cernusco Luna Nunzia V, Valentini V, De Paoli A, Maurizi ER, Lupattelli M, et al. No benefit of adjuvant Fluorouracil Leucovorin chemotherapy after neoadjuvant chemoradiotherapy in locally advanced cancer of the rectum (LARC): Long term results of a randomized trial (I-CNR-RT). Radiother Oncol 2014;113(2):223-9.

16. Bosset JF, Calais G, Mineur L, Maingon P, Stojanovic-Rundic S, Bensadoun RJ, et al. Fluorouracil-based adjuvant chemotherapy after preoperative chemoradiotherapy in rectal cancer: long-term results of the EORTC 22921 randomised study. Lancet Oncol 2014;15(2):184-90.

17. Aschele C, Cionini L, Lonardi S, Pinto C, Cordio S, Rosati G, et al. Primary tumor response to preoperative chemoradiation with or without oxaliplatin in locally advanced rectal cancer: pathologic results of the STAR-01 randomized phase III trial. J Clin Oncol 2011;29(20):277380 .

18. Azria D, Doyen J, Jarlier M, Martel-Lafay I, Hennequin C, Etienne P, et al. Late toxicities and clinical outcome at 5 years of the ACCORD 12/0405-PRODIGE 02 trial comparing two neoadjuvant chemoradiotherapy regimens for intermediate-risk rectal cancer. Ann Oncol 2017;28(10):2436-2442.

19. O'Connell MJ, Colangelo LH, Beart RW, Petrelli NJ, Allegra CJ, Sharif S, et al. Capecitabine and oxaliplatin in the preoperative multimodality treatment of rectal cancer: surgical end points from National Surgical Adjuvant Breast and Bowel Project trial R-04. J Clin Oncol 2014;32(18):1927-34.

20. Schmoll HJ, Stein A, Van Cutsem E, Price T, Hofheinz RD, Nordlinger B, et al. Pre- and Postoperative Capecitabine Without or With Oxaliplatin in Locally Advanced Rectal Cancer: PETACC 6 Trial by EORTC GITCG and ROG, AIO, AGITG, BGDO, and FFCD. J Clin Oncol 2021;39(1):17-29.

21. Paun BC, Cassie S, MacLean AR, Dixon E, Buie WD. Postoperative complications following surgery for rectal cancer. Ann Surg 2010;251(5):807-18. 
22. Hendren SK, O'Connor BI, Liu M, Asano T, Cohen Z, Swallow CJ, et al. Prevalence of male and female sexual dysfunction is high following surgery for rectal cancer. Ann Surg 2005;242(2):212-23.

23. Bowel function 14 years after preoperative short-course radiotherapy and total mesorectal excision for rectal cancer: report of a multicenter randomized trial. Chen TY, Wiltink LM, Nout RA, Meershoek-Klein Kranenbarg E, Laurberg S, Marijnen CAM, et al. Clin Colorectal Cancer 2015;14(2):106-114.

24. Wiltink LM, Nout RA, van der Voort van Zyp JR, Ceha HM, Fiocco M, et al. Long-Term Health-Related Quality of Life in Patients With Rectal Cancer After Preoperative Short-Course and Long-Course (Chemo) Radiotherapy. Clin Colorectal Cancer. 2016 Sep;15(3):e93-9.

25. Adam JP, Denost Q, Capdepont M, van Geluwe B, Rullier E. Prospective and Longitudinal Study of Urogenital Dysfunction After Proctectomy for Rectal Cancer. Dis Colon Rectum 2016;59(9):822-830.

26. Habr-Gama A, Perez RO, Nadalin W, Sabbaga J, Ribeiro U Jr, Silva e Sousa AH Jr, et al. Operative versus nonoperative treatment for stage 0 distal rectal cancer following chemoradiation therapy: long-term results. Ann Surg 2004;240(4):711-7.

27. Piessen G, Cabral C, Benoist S, Penna C, Nordlinger B. Previous transanal full-thickness excision increases the morbidity of radical resection for rectal cancer. Colorectal Dis 2012;14(4):445-452.

28. Rullier E, Rouanet P, Tuech JJ, Valverde A, Lelong B, Rivoire M, et al. Organ preservation for rectal cancer (GRECCAR 2): a prospective, randomised, open-label, multicentre, phase 3 trial. Lancet 2017;390(10093):469-479.

29. Bahadoer RR, Dijkstra EA, van Etten B, Marijnen CAM, Putter H, Kranenbarg EM, et al. Short-course radiotherapy followed by chemotherapy before total mesorectal excision (TME) versus preoperative chemoradiotherapy, TME, and optional adjuvant chemotherapy in locally advanced rectal cancer (RAPIDO): a randomised, open-label, phase 3 trial. Lancet Oncol 2020:S1470-2045(20)30555-6.

30. Conroy T, Lamfichekh N, Etienne PL, Rio E, Francois E, Mesgouez-Nebout N, et al. Total neoadjuvant therapy with mFOLFIRINOX versus preoperative chemoradiation in patients with locally advanced rectal cancer: Final results of PRODIGE 23 phase III trial, a UNICANCER GI trial. J Clin Oncol 2020;38:15_suppl:4007-4007.

31. Bonnetain F, Bosset JF, Gerard JP, Calais G, Conroy T, Mineur L, et al. What is the clinical benefit of preoperative chemoradiotherapy with 5FU/leucovorin for T3-4 rectal cancer in a pooled analysis of EORTC 22921 and FFCD 9203 trials: surrogacy in question? Eur J Cancer 2012;48(12):1781-90.

32. Valentini V, van Stiphout RG, Lammering G, Gambacorta MA, Barba MC, Bebenek M, et al. Selection of appropriate end-points (pCR vs $2 \mathrm{yDFS}$ ) for tailoring treatments with prediction models in locally advanced rectal cancer. Radiother Oncol 2015;114(3):302-9. 
33. Erlandsson J, Holm T, Pettersson D, Berglund Å, Cedermark B, Radu C, et al. Optimal fractionation of preoperative radiotherapy and timing to surgery for rectal cancer (Stockholm III): a multicentre, randomised, non-blinded, phase 3, non-inferiority trial. Lancet Oncol 2017;18(3):336-346.

34. Erlandsson J, Lörinc E, Ahlberg M, Pettersson D, Holm T, Glimelius B, et al. Tumour regression after radiotherapy for rectal cancer - Results from the randomised Stockholm III trial. Radiother Oncol 2019;135:178-186.

35. Bujko K, Wyrwicz L, Rutkowski A, Malinowska M, Pietrzak L, Kryński J, et al. Longcourse oxaliplatin-based preoperative chemoradiation versus 5 × 5 Gy and consolidation chemotherapy for cT4 or fixed cT3 rectal cancer: results of a randomized phase III study. Ann Oncol 2016;27(5):834-42.

36. Ciseł B, Pietrzak L, Michalski W, Wyrwicz L, Rutkowski A, Kosakowska E, et al. Longcourse preoperative chemoradiation versus 5 x 5 Gy and consolidation chemotherapy for clinical T4 and fixed clinical T3 rectal cancer: long-term results of the randomized Polish II study. Ann Oncol 2019;30(8):1298-1303.

37. Lefevre JH, Mineur L, Kotti S, Rullier E, Rouanet P, de Chaisemartin C, et al. Effect of Interval (7 or 11 weeks) Between Neoadjuvant Radiochemotherapy and Surgery on Complete Pathologic Response in Rectal Cancer: A Multicenter, Randomized, Controlled Trial (GRECCAR-6). J Clin Oncol 2016;34(31):3773-3780.

38. Lefèvre JH, Mineur L, Cachanado M, Denost Q, Rouanet P, de Chaisemartin C, et al. Does A Longer Waiting Period After Neoadjuvant Radio-chemotherapy Improve the Oncological Prognosis of Rectal Cancer?: Three Years' Follow-up Results of the Greccar-6 Randomized Multicenter Trial. Ann Surg 2019;270(5):747-754.

39. Bachet JB, Lucidarme O, Levache CB, Barbier E, Raoul JL, Lecomte T, et al. FOLFIRINOX as induction treatment in rectal cancer patients with synchronous metastases: Results of the FFCD 1102 phase II trial. Eur J Cancer 2018;104:108-116.

40. Koike J, Funahashi K, Yoshimatsu K, Yokomizo H, Kan H, Yamada T, et al. Efficacy and safety of neoadjuvant chemotherapy with oxaliplatin, 5-fluorouracil, and levofolinate for T3 or T4 stage II/III rectal cancer: the FACT trial. Cancer Chemother Pharmacol 2017;79(3):519525.

41. Chua YJ, Barbachano Y, Cunningham D, Oates JR, Brown G, Wotherspoon A, et al. Neoadjuvant capecitabine and oxaliplatin before chemoreadiotherapy and total mesorectal excision in MRI-defined poor-risk rectal cancer: a phase 2 trial. Lancet Oncol 2010;11(3):2418.

42. Koeberle D, Burkhard R, von Moos R, Winterhalder R, Hess V, Heitzmann F, et al. Phase II study of capecitabine and oxaliplatin given prior to and concurrently with preoperative pelvic radiotherapy in patients with locally advanced rectal cancer. Br J Cancer 2008;98(7):1204-9.

43. Fernández-Martos C, Pericay C, Aparicio J, Salud A, Safont M, Massuti B, et al. Phase II, randomized study of concomitant chemoradiotherapy followed by surgery and adjuvant capecitabine plus oxaliplatin (CAPOX) compared with induction CAPOX followed by 
concomitant chemoradiotherapy and surgery in magnetic resonance imaging-defined, locally advanced rectal cancer: Grupo cancer de recto 3 study. J Clin Oncol 2010;28(5):859-65.

44. Maréchal R, Vos B, Polus M, Delaunoit T, Peeters M, Demetter P, et al. Short course chemotherapy followed by concomitant chemoradiotherapy and surgery in locally advanced rectal cancer: a randomized multicentric phase II study. Ann Oncol 2012;23(6):1525-30.

45. Fokas E, Allgäuer M, Polat B, Klautke G, Grabenbauer GG, Fietkau R, et al. Randomized Phase II Trial of Chemoradiotherapy Plus Induction or Consolidation Chemotherapy as Total Neoadjuvant Therapy for Locally Advanced Rectal Cancer: CAO/ARO/AIO-12. J Clin Oncol 2019;37(34):3212-3222.

46. Fernandez-Martos C, Garcia-Albeniz X, Pericay C, Maurel J, Aparicio J, Montagut C, et al. Chemoradiation, surgery and adjuvant chemotherapy versus induction chemotherapy followed by chemoradiation and surgery: long-term results of the Spanish GCR-3 phase II randomized trial. Ann Oncol 2015;26(8):1722-8.

47. Garcia-Aguilar J, Chow OS, Smith DD, Marcet JE, Cataldo PA, Varma MG, et al. Effect of adding mFOLFOX6 after neoadjuvant chemoradiation in locally advanced rectal cancer: a multicentre, phase 2 trial. Lancet Oncol 2015;16(8):957-66.

48. van der Valk MJM, Marijnen CAM, van Etten B, Dijkstra EA, Hilling DE, Kranenbarg EM, et al. Compliance and tolerability of short-course radiotherapy followed by preoperative chemotherapy and surgery for high-risk rectal cancer - Results of the international randomized RAPIDO-trial. Radiother Oncol 2020;147:75-83.

49. Glynne-Jones R, Hughes R. Critical appraisal of the 'wait and see' approach in rectal cancer for clinical complete responders after chemoradiation. Br J Surg 2012;99(7):897-909.

50. van der Valk MJM, Hilling DE, Bastiaannet E, Meershoek-Klein Kranenbarg E, Beets GL, Figueiredo NL, et al. Long-term outcomes of clinical complete responders after neoadjuvant treatment for rectal cancer in the International Watch \& Wait Database (IWWD): an international multicentre registry study. Lancet 2018;391(10139):2537-2545.

51. Dattani M, Heald RJ, Goussous G, Broadhurst J, Sao Juliao G, Habr-Gama A, et al. Oncological and survival outcomes in watch and wait patients with a clinical complete response after neoadjuvant chemoradiotherapy for rectal cancer: a systematic review and pooled analysis. JAMA Surg 2018;268(6):955-967

52. Garcia-Aguilar J, Patil S, Kim JK, Yuval JB, Thompson H, Verheij F, et al. Preliminary results of the organ preservation of rectal adenocarcinoma (OPRA) trial. J Clin Oncol 2020 38:15_suppl, 4008-4008.

53. Garcia-Aguilar J, Renfro LA, Chow OS, Shi Q, Carrero XW, Lynn PB, et al. Organ preservation for clinical T2N0 distal rectal cancer using neoadjuvant chemoradiotherapy and local excision (ACOSOG Z6041): results of an open-label, single-arm, multi-institutional, phase 2 trial. Lancet Oncol 2015;16(15):1537-1546.

54. Stijns RCH, de Graaf EJR, Punt CJA, Nagtegaal ID, Nuyttens JJME, van Meerten E, et al. Long-term Oncological and Functional Outcomes of Chemoradiotherapy Followed by Organ- 
Sparing Transanal Endoscopic Microsurgery for Distal Rectal Cancer: The CARTS Study. JAMA Surg 2019;154(1):47-54.

55. Rullier E, Vendrely V, Asselineau J, Rouanet P, Tuech JJ, Valverde A, et al. Organ preservation with chemoradiotherapy plus local excision for rectal cancer: 5-year results of the GRECCAR 2 randomised trial. Lancet Gastroenterol Hepatol 2020;5(5):465-474.

56. Fernandez LM, São Julião GP, Figueiredo NL, Beets GL, van der Valk MJM, Bahadoer $\mathrm{RR}$, et al. Conditional recurrence-free survival of clinical complete responders managed by watch and wait after neoadjuvant chemoradiotherapy for rectal cancer in the International Watch \& Wait Database: a retrospective, international, multicentre registry study. Lancet Oncol 2021;22(1):43-50.

57. Schrag D, Weiser MR, Goodman KA, Gonen M, Hollywood E, Cercek A, et al. Neoadjuvant chemotherapy without routine use of radiation therapy for patients with locally advanced rectal cancer: a pilot trial. J Clin Oncol 2014;32(6):513-8.

58. Deng Y, Chi P, Lan P, Wang L, Chen W, Cui L, et al. Neoadjuvant Modified FOLFOX6 With or Without Radiation Versus Fluorouracil Plus Radiation for Locally Advanced Rectal Cancer: Final Results of the Chinese FOWARC Trial. J Clin Oncol 2019;37(34):3223-3233. 


\section{Essai PRODIGE 23}

Cancer du rectum T34 et/ou N+

Pôle inférieur $<15 \mathrm{~cm}$ de la marge anale

Indice de performance ECOG 0-1

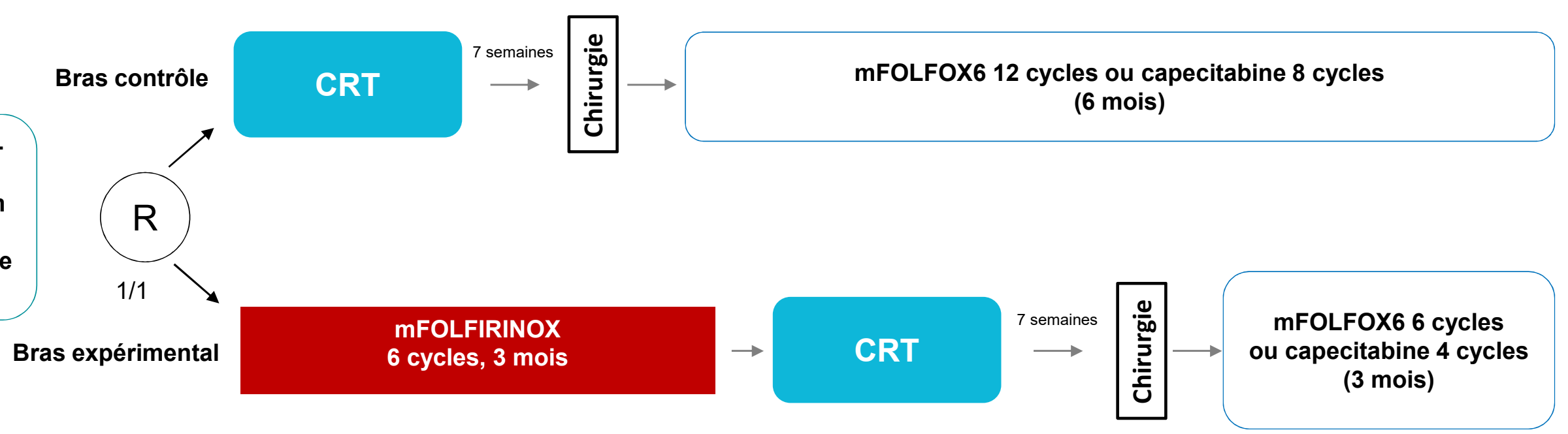

Critère de jugement principal : survie sans récidive 


\section{Essai RAPIDO}

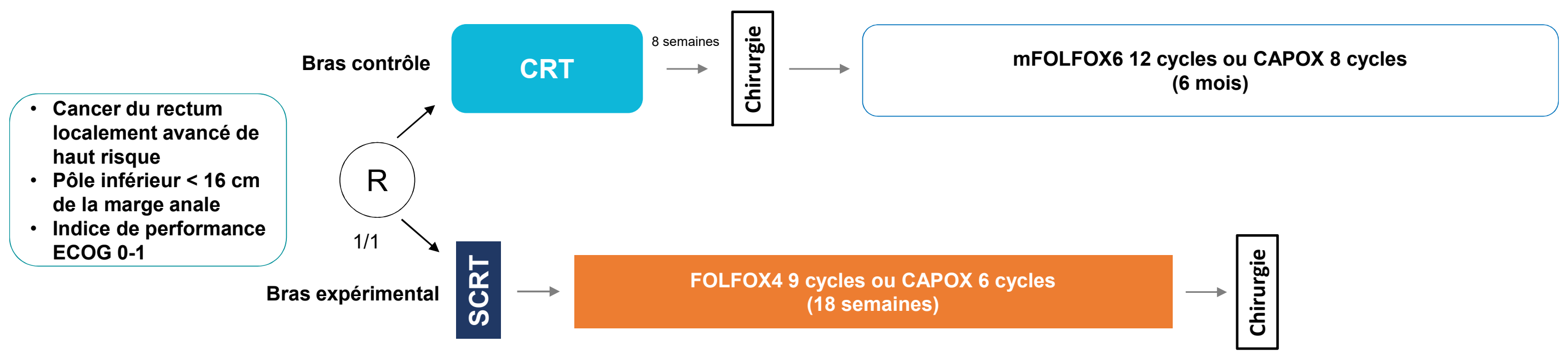

Critère de jugement principal : échec du traitement lié à la maladie 
Tableau 1 : Taux de réponse pathologique complète (RPc) en intention de traiter dans les études de phase 2-3 récentes

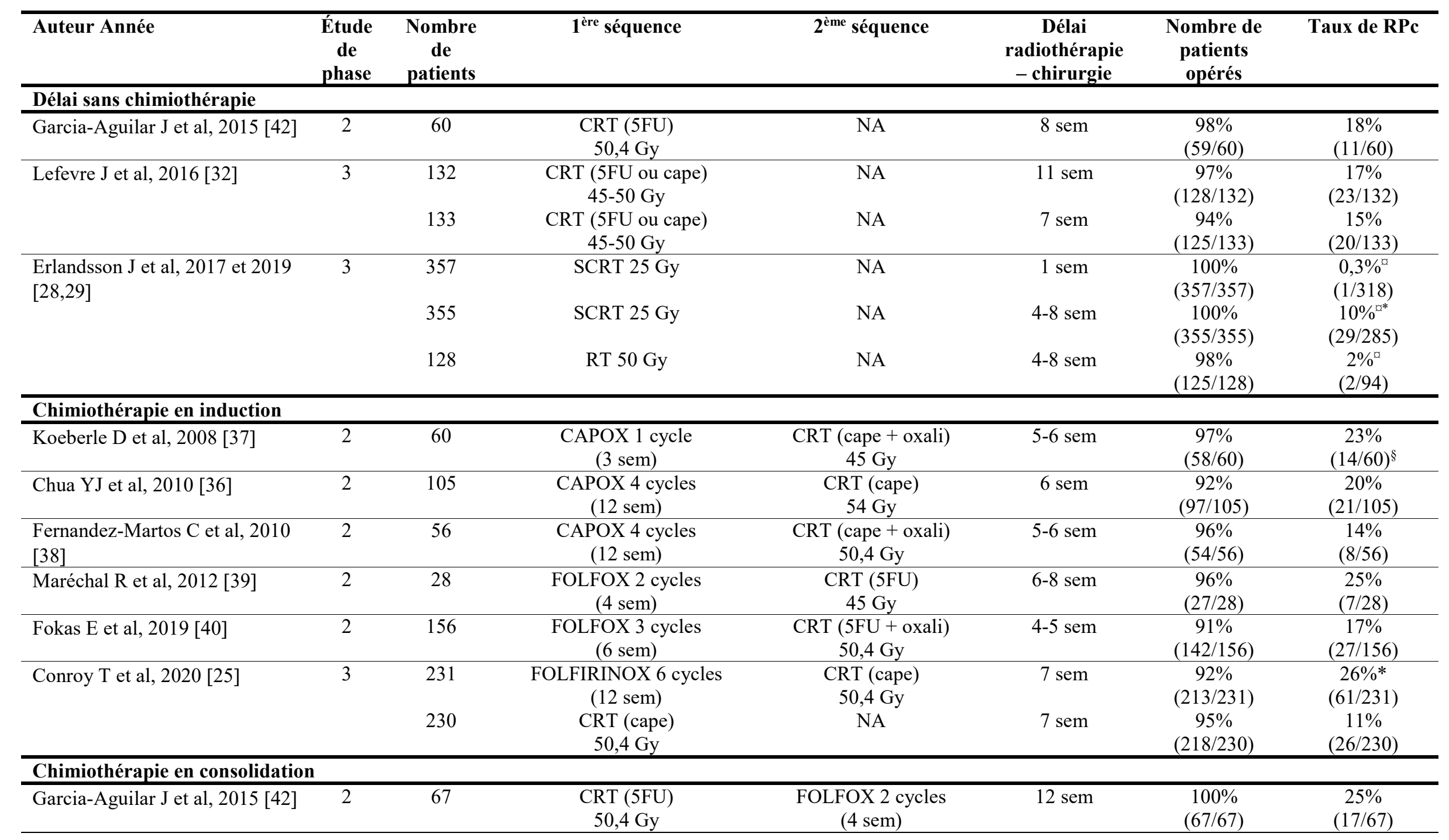




\begin{tabular}{|c|c|c|c|c|c|c|c|}
\hline & & $\begin{array}{l}67 \\
65\end{array}$ & $\begin{array}{c}\text { CRT (5FU) } \\
50,4 \text { Gy } \\
\text { CRT (5FU) } \\
50,4 \text { Gy }\end{array}$ & $\begin{array}{l}\text { FOLFOX } 4 \text { cycles } \\
\quad(8 \text { sem }) \\
\text { FOLFOX } 6 \text { cycles } \\
(12 \text { sem })\end{array}$ & $\begin{array}{l}16 \mathrm{sem} \\
20 \mathrm{sem}\end{array}$ & $\begin{array}{c}96 \% \\
(64 / 67) \\
100 \% \\
(65 / 65) \\
\end{array}$ & $\begin{array}{c}30 \% \\
(30 / 67) \\
38 \% * \\
(25 / 67) \\
\end{array}$ \\
\hline Fokas E et al, 2019 [40] & 2 & 150 & $\begin{array}{c}\text { CRT (5FU + oxali) } \\
50,4 \text { Gy }\end{array}$ & $\begin{array}{l}\text { FOLFOX } 3 \text { cycles } \\
(6 \mathrm{sem})\end{array}$ & 13-14 sem & $\begin{array}{c}95 \% \\
(142 / 150)\end{array}$ & $\begin{array}{c}25 \% \\
(38 / 150)\end{array}$ \\
\hline $\begin{array}{l}\text { Bujko K et al, } 2016 \text { [30] } \\
\text { Cisel B et al, } 2019 \text { [31] }\end{array}$ & 3 & $\begin{array}{l}261 \\
254\end{array}$ & $\begin{array}{c}\text { SCRT } 25 \text { Gy } \\
\text { CRT (5FU + oxali) } \\
50,4 \text { Gy }\end{array}$ & $\begin{array}{c}\text { FOLFOX } 3 \text { cycles } \\
\text { (6 sem) } \\
\text { NA }\end{array}$ & $\begin{array}{l}11 \mathrm{sem} \\
7 \mathrm{sem}\end{array}$ & $\begin{array}{c}84 \% \\
(41 / 261) \\
81 \% \\
(49 / 254) \\
\end{array}$ & $\begin{array}{c}14 \% \\
(37 / 261) \\
9 \% \\
(24 / 254) \\
\end{array}$ \\
\hline Bahadoer RR et al, 2020 [24] & 3 & $\begin{array}{l}462 \\
450\end{array}$ & $\begin{array}{l}\text { SCRT } 25 \text { Gy } \\
\text { CRT (cape) } \\
50,4 \text { Gy }\end{array}$ & $\begin{array}{c}\text { CAPOX or FOLFOX } \\
(18 \mathrm{sem}) \\
\text { NA }\end{array}$ & 22-24 sem & $\begin{array}{c}92 \% \\
(426 / 462) \\
89 \% \\
(400 / 450)\end{array}$ & $\begin{array}{c}26 \% * \\
(120 / 462) \\
13 \% \\
(57 / 450)\end{array}$ \\
\hline
\end{tabular}

Abréviations : CRT : chimioradiothérapie ; RT : radiothérapie ; SCRT : radiothérapie courte ; Cape : capecitabine ; oxali : oxaliplatine ; sem : semaines ; NA : non applicable "données de pCR non analysées en intention de traiter

$\S$ qq cellules tumorales résiduelles chez 7 des 14 patients mais définis comme une réponse pathologique complète

* différence statistiquement significative 
Tableau 2 : Principales caractéristiques des patients inclus et déroulement des traitements dans les essais PRODIGE 23 et RAPIDO.

\begin{tabular}{|c|c|c|c|c|}
\hline & \multicolumn{2}{|c|}{ PRODIGE 23} & \multicolumn{2}{|c|}{ RAPIDO } \\
\hline & Bras Ex & Bras C & Bras Ex & Bras C \\
\hline \multicolumn{5}{|l|}{ Caractéristiques à l'inclusion } \\
\hline Nombre de patients & 231 & 230 & 462 & 450 \\
\hline Age médian (années) & 61 & 62 & 62 & 62 \\
\hline Sexe masculin & $65 \%$ & $68 \%$ & $65 \%$ & $69 \%$ \\
\hline \multicolumn{5}{|l|}{ OMS/ECOG PS } \\
\hline 0 & $78 \%$ & $81 \%$ & $80 \%$ & $79 \%$ \\
\hline 1 & $22 \%$ & $19 \%$ & $20 \%$ & $21 \%$ \\
\hline \multicolumn{5}{|l|}{ Distance de la marge anale } \\
\hline$<5 \mathrm{~cm}$ & $38 \%$ & $36 \%$ & $22 \%$ & $26 \%$ \\
\hline $5-10 \mathrm{~cm}$ & $49 \%$ & $51 \%$ & $39 \%$ & $34 \%$ \\
\hline$>10 \mathrm{~cm}$ & $13 \%$ & $13 \%$ & $32 \%$ & $34 \%$ \\
\hline non renseignée & - & - & $7 \%$ & $7 \%$ \\
\hline Stade $4^{*}$ & $18 \%$ & $16 \%$ & $32 \%$ & $30 \%$ \\
\hline Stade $\mathrm{N}+{ }^{*}$ & $89 \%$ & $90 \%$ & $91 \%$ & $92 \%$ \\
\hline MRC envahie & $26 \%$ & $28 \%$ & $62 \%$ & $60 \%$ \\
\hline \multicolumn{5}{|l|}{ Traitements reçus $^{\beta}$} \\
\hline FOLFIRINOX 6 cycles & $90 \%$ & - & - & - \\
\hline SCRT & - & - & $99 \%$ & - \\
\hline CAPOX 6 cycles ou FOLFOX 9 cycles & - & - & $85 \%$ & - \\
\hline CRT & $95 \%$ & $99 \%$ & - & $98 \%$ \\
\hline Chirurgie & $92 \%$ & $95 \%$ & $92 \%$ & $89 \%$ \\
\hline Chimiothérapie adjuvante & $70 \%$ & $69 \%$ & - & $42 \%$ \\
\hline
\end{tabular}

Abréviations : Bras Ex : bras expérimental ; Bras C : bras contrôle ; PS : indice de performance ; MRC : marge de résection circonférentielle envahie; SCRT, radiothérapie courte; CRT, chimioradiothérapie.

${ }^{*}$ au diagnostic ; ${ }^{\beta}$ en intention de traiter 
Tableau 3 : Principaux résultats oncologiques des essais PRODIGE 23 et RAPIDO en intention de traiter.

\begin{tabular}{lcccc}
\hline & \multicolumn{2}{c}{ PRODIGE 23 } & \multicolumn{2}{c}{ RAPIDO } \\
& Bras Ex & Bras C & Bras Ex & Bras C \\
Nombre de patients & 231 & 230 & 462 & 450 \\
\hline Chirurgie & & & & \\
Chirurgie à visée curative & $92 \%$ & $93 \%$ & $92 \%$ & $94 \%$ \\
$\quad$ Résection antérieure & $73 \%$ & $70 \%$ & $58 \%$ & $55 \%$ \\
Résection inter-sphinctérienne & $6 \%$ & $10 \%$ & - & - \\
$\quad$ Amputation abdominopérinéale & $13 \%$ & $13 \%$ & $34 \%$ & $39 \%$ \\
$\quad$ Intervention de Hartmann & - & - & $5 \%$ & $3 \%$ \\
Pathologie & & & & \\
Exérèse complète du mésorectum & $79 \%$ & $81 \%$ & $78 \%$ & $85 \%$ \\
Résection R0 & $86 \%$ & $88 \%$ & $83 \%$ & $80 \%$ \\
Stade ypT0 & $26 \% *$ & $12 \%$ & $28 \% *$ & $15 \%$ \\
Stade ypN0 & $76 \% *$ & $64 \%$ & $69 \% *$ & $61 \%$ \\
Réponse pathologique complète & $26 \% *$ & $11 \%$ & $26 \% *$ & $13 \%$ \\
Suivi & & & & \\
Taux de SSM ou SETLM à 3 ans & $75,7 \% *$ & $68,5 \%$ & $76,3 \% *$ & $69,6 \%$ \\
Taux de SSMeta à 3 ans & $78,8 \% *$ & $71,7 \%$ & $80,0 \% *$ & $73,2 \%$ \\
Récidive loco-régionale & $4,8 \%$ & $7,0 \%$ & $8,3 \%$ & $6,0 \%$ \\
Taux de SG à 3 ans & $91 \%$ & $88 \%$ & $89 \%$ & $89 \%$ \\
\hline
\end{tabular}

Abréviations : Bras Ex : bras expérimental ; Bras C : bras contrôle ; SSM : survie sans maladie ; SETLM : survie sans échec du traitement lié à la maladie; SSMeta : survie sans métastase

* Différence statistiquement significative 